\title{
MCM Paradox: Abundance of Eukaryotic Replicative Helicases and Genomic Integrity
}

\author{
Mitali Das, ${ }^{1}$ Sunita Singh, ${ }^{2}$ Satyajit Pradhan, ${ }^{3}$ and Gopeshwar Narayan ${ }^{1}$ \\ ${ }^{1}$ Cancer Genetics Laboratory, Department of Molecular and Human Genetics, Banaras Hindu University, Varanasi, India \\ ${ }^{2}$ Department of Zoology, Mahila Mahavidyalaya College, Banaras Hindu University, Varanasi, India \\ ${ }^{3}$ Department of Radiotherapy \& Radiation Medicine, Banaras Hindu University, Varanasi, India
}

Correspondence should be addressed to Gopeshwar Narayan; gnarayan@bhu.ac.in

Received 29 August 2014; Accepted 30 September 2014; Published 19 October 2014

Academic Editor: Malayannan B. Subramaniam

Copyright (C) 2014 Mitali Das et al. This is an open access article distributed under the Creative Commons Attribution License, which permits unrestricted use, distribution, and reproduction in any medium, provided the original work is properly cited.

\begin{abstract}
As a crucial component of DNA replication licensing system, minichromosome maintenance (MCM) 2-7 complex acts as the eukaryotic DNA replicative helicase. The six related MCM proteins form a heterohexamer and bind with ORC, CDC6, and Cdt1 to form the prereplication complex. Although the MCMs are well known as replicative helicases, their overabundance and distribution patterns on chromatin present a paradox called the "MCM paradox." Several approaches had been taken to solve the MCM paradox and describe the purpose of excess MCMs distributed beyond the replication origins. Alternative functions of these MCMs rather than a helicase had also been proposed. This review focuses on several models and concepts generated to solve the MCM paradox coinciding with their helicase function and provides insight into the concept that excess MCMs are meant for licensing dormant origins as a backup during replication stress. Finally, we extend our view towards the effect of alteration of MCM level. Though an excess MCM constituent is needed for normal cells to withstand stress, there must be a delineation of the threshold level in normal and malignant cells. This review also outlooks the future prospects to better understand the MCM biology.
\end{abstract}

\section{Introduction}

In early 1980s, minichromosome maintenance (MCM) proteins were first identified in budding yeast Saccharomyces cerevisiae [1]. These mutant variants were defective in maintenance of minichromosome, a plasmid with a cloned centromere and replication origin [2, 3]. MCM proteins were thus found to have essential roles in DNA replication $[4,5]$. Subsequent identification of proteins with a particular identical sequence termed "MCM box" [6] leads to the constitution of a protein family, the MCM family. The family consists of at least 6 homologues, MCM 2-7 [1], which is directly involved in eukaryotic DNA replication [7]. MCMs remain at the centre of interest of biochemists, geneticists, and cancer biologists since 30 years of their identification. The studies provide good knowledge of MCM structure, function, particular role in DNA replication, and abnormalities leading to cancer. On the other hand, some paradoxes came forward with the advancement of studies regarding the function of MCMs in DNA replication. It has been found that the MCM hexamers are loaded in excess onto chromatin and distributed on the locations distant from the origins. Solving the paradox known as the "MCM paradox" is challenging to the scientific community. In this review we have discussed the role of MCMs in DNA replication, concepts generated to solve the paradoxes, their extension to cancer biology, and future perspective.

\section{MCMs as a Component of Replication Origins}

MCM 2-7 is expressed in all eukaryotes [1]. Physical interaction between MCM 2-7 components was determined by several molecular techniques such as two-hybrid, coimmunoprecipitation and biochemical purification [8-11]. It has been proposed that MCM 2-7 makes a heterohexameric complex in $1: 1: 1: 1: 1: 1$ ratio. The core of MCM 2-7 complex comprising MCM 4, 6, 7 has DNA helicase activity [12-14]. The other three components of the complex may play other roles 
during replication such as MCM2 binds to histones and facilitate histone redeposition during DNA synthesis [15]. However, the fact that MCM proteins are essential in vertebrate DNA replication was confirmed by the studies in Xenopus, where MCM depleted egg extracts were unable to support further DNA synthesis [16, 17].

A parallel concept of "replication licensing" was emerging in 1980s from the observation that sperm chromatin in a nucleus replicated only once in a cell cycle and became competent again in mitosis but a specific DNA sequence is not required for replication initiation. This concept hypothesized that there must be a positive "license" to mark the replicated sequences from the unreplicated ones thus preventing the reinitiation of replication at the already replicated sites [18]. The cell cycle dependent change in subcellular localization of MCMs in yeast and Xenopus proposed these proteins to be replication licensing factors $[8,15,19]$. However, the replication licensing mechanism involves a series of events and a number of proteins. The first step of replication licensing mechanism is the formation of prereplication complex (pre$\mathrm{RC})$. Pre-RC formation involves assembly of a number of proteins such as origin recognition complex (ORC), Cdc6, Cdt1, and MCM 2-7. This in turn leads to a chain of events allowing binding of DNA polymerase and other several factors to chromatin to start DNA replication [7, 20-22].

\section{MCMs Function as Helicases}

After the identification of MCMs as replication licensing factors, their biochemical function at the chromatin had been searched for. The heterohexameric ring structure of MCM 27 suggests that this protein complex may work as replicative helicase. The entire MCM 2-7 complex in fission yeast or $\operatorname{MCM}(4,6,7)_{2}$ complex from the mouse shows a globular structure with a central channel of 3-4 nm similar to many multisubunit helicases from all kingdom of life. This channel can encircle either single or double stranded DNA [1]. In 2001, Labib and Diffley [23] enquired whether MCM 2-7 is the eukaryotic DNA replication fork helicase. The first evidence came from the study of Yukio Ishimi, where they had shown that purified MCM 4, 6, 7 complexes have $3^{\prime} \rightarrow 5^{\prime}$ DNA unwinding capacity [24]. Consequently, they have also shown that mouse MCM 4, 6, 7 complexes have intrinsic DNA helicase activity [12]. Not only the MCM 4, 6, 7 subcomplex but a whole MCM 2-7 complex had been reported to have in vitro helicase activity where the MCM 2-5 junction acts as an ATP drive gap or "gate" [25]. Recent studies have shown that the MCM 2-7 proteins make a core of the replicative DNA helicase and that they are first loaded at replication origins in an inactive form. The helicase is then activated by recruitment of the Cdc45 and GINS proteins into a holo-helicase known as CMG (Cdc45, MCM 2-7, GINS) [26]. Identification of several interacting partners and the real action mechanism of this holo-helicase keeps researchers involved in studies of MCMs. Very recently it has been shown that the human CMG complex (hCMG) can unwind DNA duplex regions up to $500 \mathrm{bp}$ hydrolyzing ATP and in combination with human DNA polymerase $\varepsilon$ leads to the formation of oligonucleotide products up to $>10 \mathrm{~kb}$ [27]. The same group has also identified human chromosome transmission fidelity 4 (hCTF4) as an interacting partner of hCMG complex [28].

The helicase activity of MCM proteins was also found in archeal species [29]. Methanobacterium thermoautotrohicum, the bacterium, contains only one MCM protein which forms a homohexameric ring. The homohexamer contains a positively charged central channel of 2-4 $\mathrm{nm}$ to bind with DNA and act as a helicase [30-33]. A single MCM homolog from Sulfolobus solfataricus has been reported to form hexamers in solution and can dissolve $5^{\prime}$ tailed oligonucleotides by its helicase activity [34].

MCM 2-7 proteins are categorized in superfamily 6 (SF6) helicases $[35,36]$ in which each subunit of a hexameric helicase contains an AAA+ domain. In these helicases the ATPase sites are generated in between two adjacent subunits: one is providing the walker A and walker B motif for ATP binding and the other partner provides the arginine finger motif for ATP hydrolysis. MCM 2-7 is also related to viral homohexameric helicases (superfamily 3 (SF3)) including the SV40 large $\mathrm{T}$ antigen and papilloma virus E1 protein [26].

Boos et al. [26] also summarized the evidence, indicating MCM 2-7 as the engine of the replicative helicase. Firstly, MCM 2-7 travels with replication forks, forming part of the purified replisome progression complex (RPC) in yeast [36, 37] and vertebrates [38-41]. Secondly, acute inactivation of MCM 2-7 subunits during $S$ phase results in rapid replisome inactivation $[42,43]$ and, third, purified yeast MCM 2-7 complex shows in vitro helicase activity [25].

\section{The MCM Paradox}

Above discussed lines of evidence substantially show that MCM 2-7 complex has helicase activity to unwind double stranded DNA and thus helps to initiate DNA replication. But there are some issues that prohibit accepting the role of MCM 2-7 as helicase. The issues termed as "MCM paradox" came from two observations: (a) eukaryotic MCM 2-7 complexes are not localized at the sites of replication like viral and bacterial helicases. MCM 2-7 complexes in higher eukaryotes are distributed over nonreplicated DNA rather than on replication forks; (b) the paradox is elaborated by another observation that excess MCM heterohexamers are loaded onto chromatin rather than replication origins and ORCs [44]. The basic questions were how MCM 2-7 complexes perform their function as helicase from a distance away from the replication sites and what the purpose of the excess bound MCMs onto chromatin more than the origins themselves is.

Madine et al. [45] have shown by immunofluorescence that Xenopus MCM3 does not colocalize with sites of DNA replication. The protein is almost uniformly distributed on chromatin and is suddenly lost during replication. Same line of evidence in human cells came from another study in 1996. The authors had used high resolution confocal microscopy to determine the subnuclear sites of chromatin-bound MCM proteins in comparison to the sites of replicating DNA. They found MCM proteins to be entirely nuclear in HeLa cells at interphase and to exist in nucleoplasmic and chromatin bound forms. The chromatin bound form is present 
throughout the nucleus in late G1 and early $\mathrm{S}$ and at discrete subnuclear sites following progression into $\mathrm{S}$ phase. Interestingly, as the $S$ phase proceeds further, the MCMs are displaced from their site on replicating chromatin. Furthermore, the chromatin bound fraction was found to be absent in G2 and mitotic nuclei. The observation that MCMs are present on unreplicated chromatin rather than replicating one emphasized their role in monitoring the unreplicated chromatin to ensure only a single round of DNA replication [46]. In Chinese hamster ovary (CHO) fibroblasts, MCM2 associates with early and late replicating chromatin in late telophase. Subsequently, more MCM2 is associated to chromatin throughout the G1 phase and the maximum loading occurs at the G1/S transition. MCM2 was found to get dissociated from replicons shortly after their initiation [47]. In 2005, Mašata et al. [48] used a robust statistical method to be more accurate in analyzing the localization of MCM proteins and replication machineries. In HeLa cells, majority of MCM2 proteins do not colocalize with replication foci, but a very small but significant fraction of MCM2 signals colocalize with DNA replication foci throughout the $S$ phase. The colocalization of MCM2 protein with replication foci during $S$ phase thus explains an active function of MCM 2-7 complex in replication. Supporting the above findings, a recent in vivo licensing assay has proved that the concentration of chromatin bound MCM2 and MCM4 changes with progression of G1 phase and they fail to colocalize with other replication machinery [49].

Eukaryotic DNA replication starts at multiple sites throughout the genome. In budding yeast, the initiation sites, known as autonomously replicating sequences, are distributed all over the genome at an interval of every $40 \mathrm{~kb}$. In somatic cells, replication can be initiated once every $150 \mathrm{~kb}$. In embryonic cells of Xenopus laevis, DNA replication initiation sites are regularly spaced at every $9-12 \mathrm{~kb}$ without sequence specificity [50]. In all cases the initiation is first marked by the binding of ORC [7]. As noted earlier, the second part of MCM paradox concerns the abundance of chromatin bound ORCs and MCM 2-7 complexes. In yeast, Xenopus, and highly proliferating human cancer cells HeLa, the number of MCM 2-7 complexes bound to chromatin highly exceeds the number of origins [10,51-55]. In Xenopus, the number of MCM 2-7 complexes is found to be 10-40 times more than the actual initiation events are [56, 57]. More precisely, in Xenopus, the minimum length of DNA required to recruit ORC and MCM 2-7 is $\sim 80 \mathrm{bp}$. The ratio of MCM $2-7$ : ORC in this small fragment is perfectly $1: 1$. With a DNA fragment longer than $80 \mathrm{bp}$, the MCM 2-7: ORC ratio increased up to $40: 1$ [58].

\section{Proposed Models to Resolve "MCM Paradox"}

Several plausible mechanisms have been proposed to explain the function of MCM 2-7 as replication helicase from a distance away from the replication site. According to "MCM eviction" mechanism, there is always a minor MCM population that escapes immunodetection but is associated at replication forks and the rest major fraction is released from the replication forks upon replication initiation $[47,59]$, as a consequence of chromatin condensation [60]. A rotary pump model depicts that those MCM 2-7 complexes have always been loaded at a distance from an active replication fork and, like rotary motors, they translocate DNA along its axis by helical rotation causing unwinding at the constrained replication fork [61]. With little variation of rotary pump model, a ploughshare model has been proposed, where MCM helicase encircles and translocates on dsDNA. Unwinding of DNA double strand occurred through a rigid "ploughshare" present at the trailing edge of the helicase that is inserted in between DNA double strands and cleaves the duplex. The model also assumes that multiple pairs of MCM 2-7 hexamers bind to each replicon in G1 phase but only a single pair is activated to perform as helicase [62]. All the models have a fact in common that there must be at least a few active MCMs at the replication fork. The fraction of these MCMs at replication forks may be undetectable in comparison to the detectable fraction present on chromatin distant from the active replication sites. Together with the ideas and observations, the current model of MCM action has been proposed by Aparicio et al. [59]. The group found that, in HeLa cell nuclei, the MCM proteins, at G1 phase, first concentrate on chromatin structures that subsequently become replication forks at $S$ phase. The concentration of bound MCMs at replication forks gets reduced after replication initiation leaving a very small but detectable fraction still bound to the forks during the $S$ phase, supposed to be the helicase. The findings were similar to those of a previous study [63]. The authors proposed an extension of "MCM eviction" mechanism of MCM dynamics based on the "loop model" of mammalian replication factories [59, 64]. According to this model, at a very early onset of G1, the MCM 2-7 complexes start to be loaded onto origin DNA in an ORC-Cdc6-Cdt1-dependent manner and spread all over the adjacent sequences. The amount of MCMs to be loaded on each origin may differ influencing their firing ability. At G1-S boundary, only a few MCM 2-7 complexes get activated by Cdc45, GINS, and other protein factors. These activated MCMs become able to escape the posttranslational modification signals that can cause their dissociation from chromatin and remain bound to both active and dormant origins upon initiation of DNA synthesis. In a multireplicon chromatin arrangement, several replicon units remain organized in cohesion-stabilized loops to form a rosette structure. Most of the origins are distributed at the base of the loops confining replication event at the central part of the rosette. The activation of replication forks leads to the MCM-BP mediated dissociation of inactive MCMs [65] from chromatin and continuous entry of PCNA. A small fraction of MCMs remain always bound to the active site of the DNA replication. Thus, the first part of the MCM paradox has been solved [59].

\section{Excess MCMs Are Meant to Survive Replicative Stress}

The second part of the MCM paradox concerns the purpose of quantitative excess of MCMs over origins. The common 
approach was to reduce the dosage of MCMs in replicating cells and analyze the posteffects. In yeast, it has been found that depletion of MCM2 does not affect their growth rate but a $50 \%$ reduction of the dosage of MCM2 in yeast decreases the number of origins to be used per replication event. There was an indication that the excess MCM proteins may be required for successful initiation at all replication origins [10]. Instead of hampering cell cycle, depletion of MCM7 causes activation of checkpoint signalling in human cancer cells, suggesting their roles in replication checkpoint signalling or activation of replication origins at the time of incomplete DNA replication $[66,67]$. It is evident that MCMs may have an alternative role related to DNA replication; however, it remained difficult to conclude due to the lack of missing link(s). The concept of "dormant" origins has been proposed to be the missing link based on the origins that remain suppressed during normal DNA replication and can get activated and support initiation when replication forks are stalled in response to replicative stresses $[68,69]$. In Xenopus laevis, there are 10 dormant origins distributed adjacent to each active origin. Both the active and dormant origins are bound by MCM 2-7 complexes and have potential to carry out replication process. But the dormant origins remain inactivated during a normal replication event by ATR mediated signalling pathway. In a stressed replication condition, such as in presence of aphidicolin, when the ATR signalling is inhibited by caffeine, an ATR-kinase inhibitor, the dormant origins get activated and partially rescue the replication process. Consequently, RNAi-based partial depletion of MCMs in Caenorhabditis elegans does not show any observable effect on cell viability or proliferation but becomes lethally hypersensitive to a nontoxic dose of replication inhibitor hydroxyurea. These observations suggested the role of excess MCM 2-7 complex in licensing dormant origins during replication stress [68]. Extension of similar studies in human cancer cells shows that RNAi mediated MCM5 silencing allows the cells to grow at a normal rate but makes these MCM depleted cells vulnerable to any kind of replication stress such as hydroxyurea or aphidicolin. The authors suggest that excess MCM 2-7 provides the cells safeguards against replicative stress by licensing dormant origins [70]. An extensive study using siRNA against each component of MCM 2-7 complex in human cancer cell lines was concordant with the above findings. Acute downregulation of MCM2 and MCM3 permits the formation of a limited but sufficient amount of MCM 2-7 complex, allowing the cells to proliferate. But their hypersensitivity to replication stress shows increased chromosomal aberrations and eventually raises a checkpoint response to block mitotic division. The acute downregulation of MCM 4-7, on the other hand, may reduce the number of chromatin bound MCM 2-7 complexes beyond the threshold and thus becomes fatal. It has been suggested that the excess MCM 27 complexes license dormant origins as a backup mechanism to maintain genomic integrity during replication stress [71]. The consequences of attenuated MCM expression in whole animal were studied using mouse model. Chaos3 (chromosomal aberrations occurring spontaneously 3) is a murine chromosomal mutation of MCM4. The viable mutant allele MCM4 ${ }^{\text {Chaos3 }}$ produces the polypeptide with a change from wild type phenylalanine to isoleucine at residue 345 (F345I), producing a destabilized form of MCM4. MCM4 4 Chaos3/Chaos3 female mice develop mammary adenocarcinoma after 12month latency. Hence, mutant MCM4 causes cancer predisposition. The embryonic fibroblasts (MEFs) from these mutant mice showed increased rate of DNA breaks after treatment of aphidicolin [72]. MEFs, containing hypomorphic MCM $4^{\text {Chaos3/Chaos3 }}$, show approximately $40 \%$ reduction of all MCM 2-7 expression. Depletion of MCM2, -6, or -7 in these mutant cells resulted in deleterious effects such as growth retardation, inhibited cell proliferation, genomic instability, and cancer predisposition. However, heterozygous condition of MCM4 ${ }^{\text {Chaos3 }}$ with normal MCM3 rescued the normal phenotype by increased chromatin bound MCMs [73]. MEFs from MCM2 $2^{\text {IRES-CreERT2/IRES-CreERT2 }}$ mice show decreased origin use in presence of hydroxyurea and are thus hypersensitive to these replicative stresses [74]. Both of the studies indicate that in vivo a cellular high level of MCMs is required for licensing active origins and maintenance of dormant origins as a backup in distress. However, excess MCMs are not only meant to license dormant origins but also take part in recombinational repair under replication stress as reported in fission yeast [75].

It is evident that the replication licensing mechanism ensures the nonlicensing of a single new origin at the onset of $S$ phase to prevent rereplication $[76,77]$. In a subsequent $S$ phase, if the cells face accidental problems such as replication fork stalling or disassembly, they cannot be retrieved. Cells therefore keep backup licensed dormant origins which are not used normally but are used when replication is at stake $[69,78]$. So the most plausible explanation for the "MCM paradox" is that cells maintain abundant MCM 2-7 complexes distributed over the chromatin as a backup licensing mechanism to combat natural replicative stress generated during S phase (Figure 1).

\section{Effects of MCM Attenuation In Vivo}

The depleted level of MCMs has been proved to be deleterious for vertebrate systems. Positional cloning of mutated MCM5 in embryonic cells of Zebra fish leads to impaired retinal development characterized by deregulated cell cycle and increased rate of apoptosis. The mutation was applied in the differentiated cells at the third day of development, but the effect was restricted to those retinal stem cells hampering development of retina, tectum, and hindbrain. This indicates that different tissues respond differentially to MCM5 depletion [79]. Mutation in MCM4 was reported for the first time in human population in 2012. A frameshift mutation leads to the formation of truncated MCM4 protein. The mutation occurs due to the insertion of a single nucleotide prior to exon 2 thus shifting the splice acceptor site. This mutation is associated with a diseased phenotype called familial glucocorticoid deficiency characterized with poor adrenal development, short stature, and NK cell deficiency and increased DNA breakage $[80,81]$. However, there is no clear report of cancer predisposition in these patients. The consequences of MCM2 and MCM4 mutations were discussed 

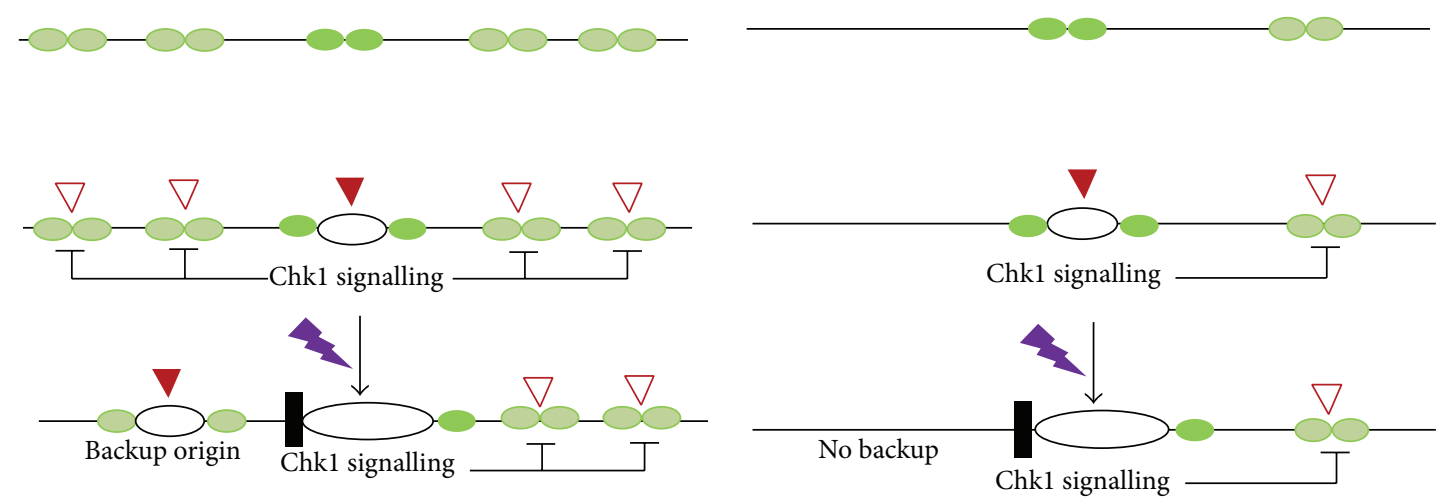

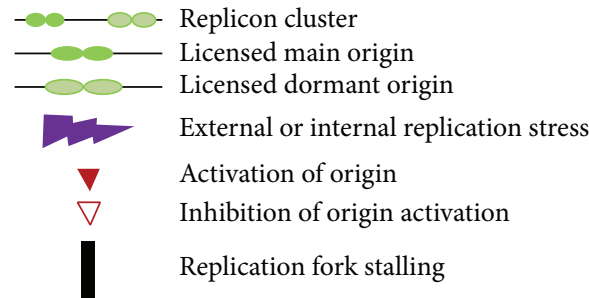

(a) Normal licensing

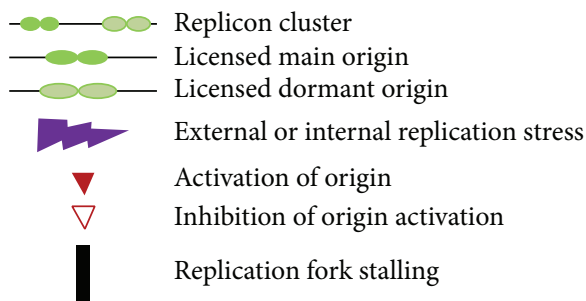

(b) Limited licensing

FIgURE 1: Purpose of excess MCMs: to license dormant origins as a backup mechanism for stress: (a) prior to S phase, the MCMs license main origins as well as many dormant origins which remain prevented to be fired by Chk1 signalling. At the time of stress, when replication forks get stalled, the backup origins get activated and continue the replication process; (b) in case of a limited licensing condition, at the time of stress there is no backup to retrieve the replication process; hence, the cells cannot withstand the stress (adapted from [71]).

in previous section [73, 74]. There is another example of dominant mutant allele $\mathrm{Mcm} 4^{\mathrm{D} 573 \mathrm{H}}$ which has been found to be associated with spontaneous dominant leukemia in mice. These leukemic mice have a normal level of MCM proteins but $\mathrm{MCM} 4^{\mathrm{D} 573 \mathrm{H}}$ is unable to incorporate into MCM 2-7 complexes causing chromosomal abnormalities leading to cancer [82]. Mouse embryos homozygous for a mutant MCM2 (Mcm2 ${ }^{\text {IRES-CreERT2/IRES-CreERT2 }}$ ) allele develop normally but can survive only for 10-12 weeks. They acquire severe impairment of tissue development due to stem cell deficiency, accumulate huge chromosomal abnormalities, and immediately die of cancer [83]. MCM2 depletion causes a high rate of thymic lymphoblastic lymphoma characterized with manifold genomic instabilities. The most common of these chromosomal abnormalities is a deletion of a genomic region of approximately $0.5 \mathrm{Mb}$ altering expression of several genes contributing to the development of cancer [84]. Above discussed citations exemplify the biochemical, cellular, and phenotypic consequences of limited licensing in the form of impeded MCM loading increased genomic instability and an ultimate formation of cancer, respectively [69].

\section{MCM Expression and Cancer}

MCMs are found to be overexpressed in multiple cancers such as in prostate cancer [85], colon cancer [86], meningioma [87], breast carcinoma [88], medulloblastoma [89], gastric adenocarcinoma [90], cervical cancer [91, 92], osteosarcoma [93], and esophageal squamous cell carcinoma [94].
Aberrant expression of individual components of MCM 27 complex makes them suitable biomarkers for malignancies [95]. MCM2 is a proposed biomarker in oligodendrioma [96], renal cell carcinoma [97], esophageal squamous cell carcinoma [98], laryngeal carcinoma [99], breast cancer [100, 101], large B cell lymphoma [102], oral cancer [103], ovarian cancer [104], and gastric cancer [105]. MCM3 is a proliferative marker in papillary thyroid carcinoma [106]. MCM4 is a proliferation marker of nonsmall cell lung cancer [107]. MCM5 is a prognostic marker of ovarian cancer and prostate cancer $[104,108]$. MCM6 protein level in plasma of hepatocellular carcinoma patients may be a novel biomarker for the malignancy [109]. Deregulated expression of MCM7 is a potential biomarker for colorectal cancer [110], small lung adenocarcinoma [111], and oral squamous cell carcinoma [112].

Hence, the overexpression of MCM genes can define the proliferative stage of a cell [113]. The reason behind deregulated expression of these proteins is still an open question. There are two possible explanations. One is that, due to an inappropriate action of CDKs, the malignant cells lost the power to differentiate, hence continuously divide and express MCMs. MCMs, in this case, are not directly oncogenic but mark the proliferative status of the cells. Another possibility is that deregulation of replication licensing system itself causes the development of cancer by inducing relicensing of replicated DNA promoting chromosomal instability [114].

Aneuploidy is an obvious consequence of inappropriate replication licensing. The overexpression of MCM2 along with other licensing proteins is significantly associated with 


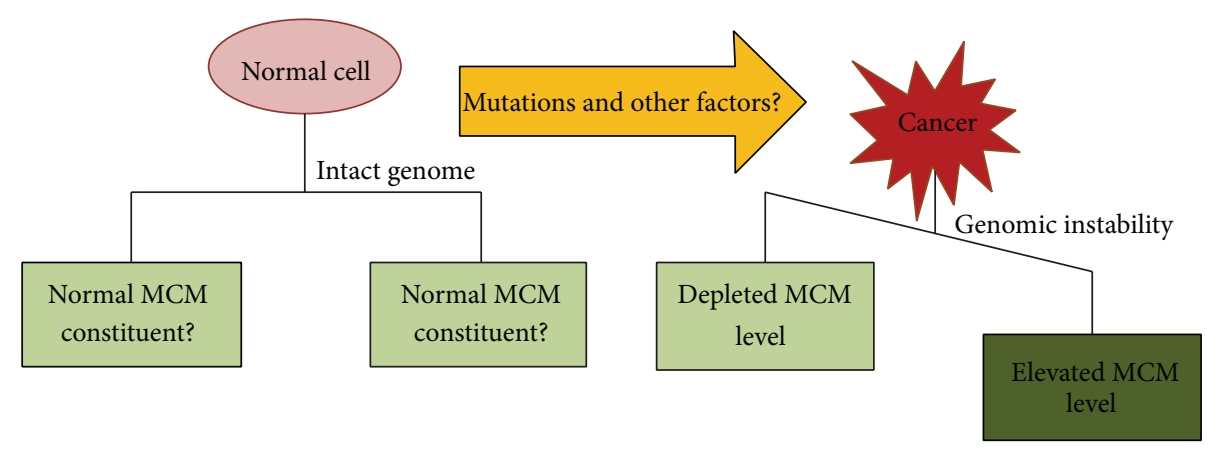

FIGURE 2: The unavoidable consequence of change in MCM level is genomic instability which may result in cancer. The threshold level of MCMs for genome maintenance of normal cells is still to be quantified and the factors causing aberrations in MCM level are yet to be identified.

aneuploidy to cause penile cancer [115]. The cervical cancer cell lines harboring high level of aneuploidy show overexpression of MCMs [92, 116]. Dosage alterations of MCMs have vivid cytogenetic background. The $3 \mathrm{q}$ and $8 \mathrm{q}$ containing MCM2 and MCM4, respectively, have been reported to show genomic amplification and chromosomal gain $[117,118]$. MCMs, thus, may cytogenetically control their expression by a positive feedback loop participating in the "vicious cycle" [119] of aneuploidy and chromosomal instability.

Inhibition of MCMs on cancer cells has also been proved to be deleterious. A geminin mediated reduction in the chromatin bound form of MCM2 promotes cell type specific checkpoint response. A p53+/Rb+ cell, U2OS, showed an early $S$ phase arrest by activation of intra- $S$ phase checkpoints. On the other hand, p53-/Rb- cell line, Saos2, showed an accumulation in late S and G2-M phase and loss of intra-S phase checkpoints. In both cases the ultimate consequence was apoptosis. In IMR90 primary fibroblasts, overexpression of geminin caused G1 arrest and did not lead to apoptosis. A "licensing checkpoint" thus may take care of the primary cells with insufficient licensed origins and prevent them from proceeding into S phase [120]. Similar results were reported where inhibition of MCM2 by RNAi produces apoptotic response in P53+ and P53- cancer cell lines but causes G1 arrest in normal cells [121]. Trichostatin A and siRNA mediated downregulation of MCM2 generated apoptotic response in HCT116 cells [122]. Knocking down MCM7 not only inhibits cell proliferation in glioblastoma multiforme tumor cells but also prevents tumor growth in mouse models of glioblastoma multiforme [123].

\section{Conclusion and Perspective}

It is evident from the above discussion that either elevation or depletion of MCM level causes genomic instability and thus consequently cancer (Figure 2). From the concept of MCM paradox and its solution we come to a conclusion that a high level of MCMs is needed by a cell normally to maintain the genomic constitution. There is a gap of knowledge as the proper quantification of the threshold level of MCMs, below or above of which is fatal, remains to be filled. Since cancer cells with elevated level of MCMs grow faster, there must be a difference in usage of dormant and main licensed origins; enlightenment in this area will help understand the replication mechanism of cancer cells better. As noted earlier that the licensing checkpoints respond differently in cancer and normal cells [119], more information is required for the complete designing of licensing checkpoint signalling. MCMs are placed downstream to many signal transduction pathways $[124,125]$ and are also reported to be controlled by miRNA $[126,127]$. It will be very interesting to record the crosstalk between several signal transduction pathways controlling MCM expression and map a complex signalling network of DNA replication licensing.

Besides DNA replication licensing, MCMs are also actively involved in transcription process. MCM 2-7 complex components are also identified as the components of RNA polymerase II transcriptional apparatus [128]. It has been demonstrated that MCM5 is indispensable as a signal transducer and activator of transcription 1 (Stat 1) mediated transcriptional activation [129]. Recently, Hubbi et al. presented another line of evidence suggesting that the abundance of MCM proteins must be meant for functions other than DNA helicase activity. They demonstrate that MCM 2, 3, 5 and MCM 7 proteins directly interact with hypoxia-inducible factor-1 (HIF-1) $\alpha$ subunit to inhibit HIF-1 transcriptional activity by oxygen dependent manner. Thus, MCM proteins play important role in regulating transcription during oxidative stress [130]. Furthermore, the existence of filamentous structure made of excess MCMs located distally from origins with a capacity of chromatin remodeling [131] extends MCM paradox towards the solution with alternative functions of MCMs in DNA metabolism.

\section{Conflict of Interests}

The authors declare that there is no conflict of interests regarding the publication of this paper.

\section{Acknowledgments}

The authors thankfully acknowledge the financial support from the Department of Biotechnology, Government of India 
(vide Project no. BT/PR9246/Med/30/17/2007), to Gopeshwar Narayan, Sunita Singh, and Satyajit Pradhan, Banaras Hindu University (Xth and XIth Plan) to Gopeshwar Narayan and Sunita Singh, and Council of Scientific and Industrial Research, Government of India, for research fellowship to Mitali Das.

\section{References}

[1] S. L. Forsburg, "Eukaryotic MCM proteins: beyond replication initiation," Microbiology and Molecular Biology Reviews, vol. 68, no. 1, pp. 109-131, 2004.

[2] G. T. Maine, P. Sinha, and B. W. Tye, "Mutants of S. cerevisiae defective in the maintenance of minichromosomes," Genetics, vol. 106, no. 3, pp. 365-385, 1984.

[3] P. Sinha, V. Chang, and B.-K. Tye, "A mutant that affects the functions of autonomously replicating sequences in yeast," Journal of Molecular Biology, vol. 192, no. 4, pp. 805-814, 1986.

[4] B. K. Tye, "Minichromosome maintenance as a genetic assay for defects in DNA replication," Methods, vol. 18, no. 3, pp. 329-334, 1999.

[5] B. K. Tye, "MCM proteins in DNA replication," Annual Review of Biochemistry, vol. 68, pp. 649-686, 1999.

[6] H. Yan, S. Gibson, and B. K. Tye, "Mcm2 and Mcm37 two proteins important for ARS activity, are related in structure and function," Genes \& Development, vol. 5, no. 6, pp. 944-957, 1991.

[7] S. P. Bell and A. Dutta, "DNA replication in eukaryotic cells," Annual Review of Biochemistry, vol. 71, pp. 333-374, 2002.

[8] J. P. J. Chong, H. M. Mahbubani, C. Y. Khoo, and J. J. Blow, "Purification of an MCM-containing complex as a component of the DNA replication licensing system," Nature, vol. 375, no. 6530, pp. 418-421, 1995.

[9] S. Dalton and B. Hopwood, "Characterization of Cdc47pminichromosome maintenance complexes in Saccharomyces cerevisiae: identification of Cdc45p as a subunit," Molecular and Cellular Biology, vol. 17, no. 10, pp. 5867-5875, 1997.

[10] M. Lei, Y. Kawasaki, and B. K. Tye, "Physical interactions among $\mathrm{Mcm}$ proteins and effects of Mcm dosage on DNA replication in Saccharomyces cerevisiae," Molecular and Cellular Biology, vol. 16, no. 9, pp. 5081-5090, 1996.

[11] Y. Adachi, J. Usukura, and M. Yanagida, "A globular complex formation by Ndal and the other five members of the MCM protein family in fission yeast," Genes to Cells, vol. 2, no. 7, pp. 467-479, 1997.

[12] Z. You, Y. Komamura, and Y. Ishimi, "Biochemical analysis of the intrinsic Mcm4-Mcm6-Mcm7 DNA helicase activity," Molecular and Cellular Biology, vol. 19, no. 12, pp. 8003-8015, 1999.

[13] Y. Ishimi and Y. Komamura-Kohno, "Phosphorylation of Mcm4 at specific sites by cyclin -dependent kinase leads to loss of Mcm4,6,7 helicase activity," Journal of Biological Chemistry, vol. 276, no. 37, pp. 34428-34433, 2001.

[14] J.-K. Lee and J. Hurwitz, "Processive DNA helicase activity of the minichromosome maintenance proteins 4, 6, and 7 complex requires forked DNA structures," Proceedings of the National Academy of Sciences of the United States of America, vol. 98, no. 1, pp. 54-59, 2001.

[15] M. Foltman, C. Evrin, G. de Piccoli et al., "Eukaryotic replisome components cooperate to process histones during chromosome replication," Cell Reports, vol. 3, no. 3, pp. 892-904, 2013.

[16] M. A. Madine, C.-Y. Khoo, A. D. Mills, and R. A. Laskey, "MCM3 complex required for cell cycle regulation of DNA replication in vertebrate cells," Nature, vol. 375, no. 6530, pp. 421-424, 1995.

[17] Y. Kubota, S. Mimura, S.-I. Nishimoto, T. Masuda, H. Nojima, and $\mathrm{H}$. Takisawa, "Licensing of DNA replication by a multiprotein complex of MCM/P1 proteins in Xenopus eggs," The EMBO Journal, vol. 16, no. 11, pp. 3320-3331, 1997.

[18] J. J. Blow, S. M. Dilworth, C. Dingwall, A. D. Mills, and R. A. Laskey, "Chromosome replication in cell-free systems from Xenopus eggs," Philosophical transactions of the Royal Society of London Series B: Biological sciences, vol. 317, no. 1187, pp. 483494, 1987.

[19] K. M. Hennessy, C. D. Clark, and D. Botstein, "Subcellular localization of yeast CDC46 varies with the cell cycle," Genes and Development, vol. 4, no. 12, pp. 2252-2263, 1990.

[20] R. Laskey, “The Croonian Lecture 2001 hunting the antisocial cancer cell: MCM proteins and their exploitation," Philosophical Transactions of the Royal Society B: Biological Sciences, vol. 360, no. 1458, pp. 1119-1132, 2005.

[21] T. J. Takara and S. P. Bell, "Multiple Cdt1 molecules act at each origin to load replication-competent Mcm2-7 helicases," The EMBO Journal, vol. 30, no. 24, pp. 4885-4896, 2011.

[22] L. Wu, Y. Liu, and D. Kong, "Mechanism of chromosomal DNA replication initiation and replication fork stabilization in eukaryotes," Science China Life Sciences, vol. 57, no. 5, pp. 482487, 2014

[23] K. Labib and J. F. X. Diffley, "Is the MCM2-7 complex the eukaryotic DNA replication fork helicase?" Current Opinion in Genetics and Development, vol. 11, no. 1, pp. 64-70, 2001.

[24] Y. Ishimi, "A DNA helicase activity is associated with an MCM4, -6 , and -7 protein complex," The Journal of Biological Chemistry, vol. 272, no. 39, pp. 24508-24513, 1997.

[25] M. L. Bochman and A. Schwacha, "The Mcm2-7 complex has in vitro helicase activity," Molecular Cell, vol. 31, no. 2, pp. 287-293, 2008.

[26] D. Boos, J. Frigola, and J. F. X. Diffley, "Activation of the replicative DNA helicase: breaking up is hard to do," Current Opinion in Cell Biology, vol. 24, no. 3, pp. 423-430, 2012.

[27] Y.-H. Kang, W. C. Galal, A. Farina, I. Tappin, and J. Hurwitz, "Properties of the human Cdc45/Mcm2-7/GINS helicase complex and its action with DNA polymerase $\varepsilon$ in rolling circle DNA synthesis," Proceedings of the National Academy of Sciences of the United States of America, vol. 109, no. 16, pp. 6042-6047, 2012.

[28] Y.-H. Kang, A. Farina, V. P. Bermudez et al., "Interaction between human Ctf4 and the Cdc45/Mcm2-7/GINS (CMG) replicative helicase," Proceedings of the National Academy of Sciences of the United States of America, vol. 110, no. 49, pp. 19760-19765, 2013.

[29] A. Costa and S. Onesti, "Structural biology of MCM helicases," Critical Reviews in Biochemistry and Molecular Biology, vol. 44, no. 5, pp. 326-342, 2009.

[30] Z. Kelman, J. K. Lee, and J. Hurwitz, "The single minichromosome maintenance protein of Methanobacterium thermoautotrophicum $\Delta \mathrm{H}$ contains DNA helicase activity," Proceedings of the National Academy of Sciences of the United States of America, vol. 96, no. 26, pp. 14783-14788, 1999.

[31] D. F. Shechter, C. Y. Ying, and J. Gautier, "The intrinsic DNA helicase activity of Methanobacterium thermoautotrophicum $\Delta \mathrm{H}$ minichromosome maintenance protein," The Journal of Biological Chemistry, vol. 275, no. 20, pp. 15049-15059, 2000. 
[32] J. P. J. Chong, M. K. Hayashi, M. N. Simon, R.-M. Xu, and B. Stillman, "A double-hexamer archaeal minichromosome maintenance protein is an ATP-dependent DNA helicase," Proceedings of the National Academy of Sciences of the United States of America, vol. 97, no. 4, pp. 1530-1535, 2000.

[33] R. J. Fletcher, B. E. Bishop, R. P. Leon, R. A. Sclafani, C. M. Ogata, and X. S. Chen, "The structure and function of MCM from archaeal M. thermoautotrophicum," Nature Structural Biology, vol. 10, no. 3, pp. 160-167, 2003.

[34] F. Carpentieri, M. de Felice, M. de Falco, M. Rossi, and F. M. Pisani, "Physical and functional interaction between the minichromosome maintenance-like DNA helicase and the singlestranded DNA binding protein from the crenarchaeon Sulfolobus solfataricus," The Journal of Biological Chemistry, vol. 277, no. 14, pp. 12118-12127, 2002.

[35] A. Y. Lyubimov, M. Strycharska, and J. M. Berger, “The nuts and bolts of ring-translocase structure and mechanism," Current Opinion in Structural Biology, vol. 21, no. 2, pp. 240-248, 2011.

[36] O. M. Aparicio, D. M. Weinstein, and S. P. Bell, "Components and dynamics of DNA replication complexes in S. cerevisiae: redistribution of MCM proteins and Cdc45p during S phase," Cell, vol. 91, no. 1, pp. 59-69, 1997.

[37] A. Gambus, R. C. Jones, A. Sanchez-Diaz et al., "GINS maintains association of Cdc45 with MCM in replisome progression complexes at eukaryotic DNA replication forks," Nature Cell Biology, vol. 8, no. 4, pp. 358-366, 2006.

[38] M. Pacek, A. V. Tutter, Y. Kubota, H. Takisawa, and J. C. Walter, "Localization of MCM2-7, Cdc45, and GINS to the site of DNA unwinding during eukaryotic DNA replication," Molecular Cell, vol. 21, no. 4, pp. 581-587, 2006.

[39] T. Aparicio, E. Guillou, J. Coloma, G. Montoya, and J. Méndez, "The human GINS complex associates with Cdc45 and MCM and is essential for DNA replication," Nucleic Acids Research, vol. 37, no. 7, pp. 2087-2095, 2009.

[40] A. Gambus, G. A. Khoudoli, R. C. Jones, and J. J. Blow, "MCM27 form double hexamers at licensed origins in Xenopus egg extract," The Journal of Biological Chemistry, vol. 286, no. 13, pp. 11855-11864, 2011.

[41] J.-S. Im, S.-H. Ki, A. Farina, D.-S. Jung, J. Hurwitz, and J.-K. Lee, "Assembly of the Cdc45-Mcm2-7-GINS complex in human cells requires the Ctf4/And-1, RecQL4, and Mcm10 proteins," Proceedings of the National Academy of Sciences of the United States of America, vol. 106, no. 37, pp. 15628-15632, 2009.

[42] K. Labib, J. A. Tercero, and J. F. X. Diffley, "Uninterrupted $\mathrm{MCH} 2-7$ function required for DNA replication fork progression," Science, vol. 288, no. 5471, pp. 1643-1647, 2000.

[43] M. Pacek and J. C. Walter, "A requirement for MCM7 and Cdc45 in chromosome unwinding during eukaryotic DNA replication," The EMBO Journal, vol. 23, no. 18, pp. 3667-3676, 2004.

[44] O. Hyrien, K. Marheineke, and A. Goldar, "Paradoxes of eukaryotic DNA replication: MCM proteins and the random completion problem," BioEssays, vol. 25, no. 2, pp. 116-125, 2003.

[45] M. A. Madine, A. D. Mills, C. Musahl, and R. A. Laskey, "The nuclear envelope prevents reinitiation of replication by regulating the binding of MCM3 to chromatin in Xenopus egg extracts," Current Biology, vol. 5, no. 11, pp. 1270-1279, 1995.

[46] T. Krude, C. Musahl, R. A. Laskey, and R. Knippers, "Human replication proteins hCdc21, hCdc46 and P1Mcm3 bind chromatin uniformly before S-phase and are displaced locally during DNA replication," Journal of Cell Science, vol. 109, no. 2, pp. 309318, 1996.
[47] D. S. Dimitrova, I. T. Todorov, T. Melendy, and D. M. Gilbert, "Mcm2, but not RPA, is a component of the mammalian early G1-phase prereplication complex," The Journal of Cell Biology, vol. 146, no. 4, pp. 709-722, 1999.

[48] M. Mašata, J. Malínský, H. Fidlerová, E. Smirnov, and I. Raška, "Dynamics of replication foci in early $S$ phase as visualized by cross-correlation function," Journal of Structural Biology, vol. 151, no. 1, pp. 61-68, 2005.

[49] I.-E. Symeonidou, P. Kotsantis, V. Roukos et al., "Multi-step loading of human minichromosome maintenance proteins in live human cells," The Journal of Biological Chemistry, vol. 288, no. 50, pp. 35852-35867, 2013.

[50] D. M. Gilbert, "Making sense of eukaryotic DNA replication origins," Science, vol. 294, no. 5540, pp. 96-100, 2001.

[51] H. Kimura, N. Nozaki, and K. Sugimoto, "DNA polymerase $\alpha$ associated protein P1, a murine homolog of yeast MCM3, changes its intranuclear distribution during the DNA synthetic period," The EMBO Journal, vol. 13, no. 18, pp. 4311-4320, 1994.

[52] R. Burkhart, D. Schulte, B. Hu, C. Musahl, F. Gohring, and R. Knippers, "Interactions of human nuclear proteins P1Mcm3 and P1Cdc46," European Journal of Biochemistry, vol. 228, no. 2, pp. 431-438, 1995.

[53] S. Donovan, J. Harwood, L. S. Drury, and J. F. X. Diffley, “Cdc6pdependent loading of $\mathrm{Mcm}$ proteins onto pre-replicative chromatin in budding yeast," Proceedings of the National Academy of Sciences of the United States of America, vol. 94, no. 11, pp. 56115616, 1997.

[54] A. Richter and R. Knippers, "High-molecular-mass complexes of human minichromosome-maintenance proteins in mitotic cells," European Journal of Biochemistry, vol. 247, no. 1, pp. 136141, 1997.

[55] M. Ritzi, M. Baack, C. Musahl, P. Romanowski, R. A. Laskey, and R. Knippers, "Human minichromosome maintenance proteins and human origin recognition complex 2 protein on chromatin," The Journal of Biological Chemistry, vol. 273, no. 38, pp. 24543-24549, 1998.

[56] H. M. Mahbubani, J. P. J. Chong, S. Chevalier, P. Thömmes, and J. J. Blow, "Cell cycle regulation of the replication licensing system: involvement of a Cdk-dependent inhibitor," The Journal of Cell Biology, vol. 136, no. 1, pp. 125-135, 1997.

[57] J. Walter and J. W. Newport, "Regulation of replicon size in Xenopus egg extracts," Science, vol. 275, no. 5302, pp. 993-995, 1997.

[58] M. C. Edwards, A. V. Tutter, C. Cvetic, C. H. Gilbert, T. A. Prokhorova, and J. C. Walter, "MCM2-7 complexes bind chromatin in a distributed pattern surrounding the origin recognition complex in Xenopus egg extracts," The Journal of Biological Chemistry, vol. 277, no. 36, pp. 33049-33057, 2002.

[59] T. Aparicio, D. Megías, and J. Méndez, "Visualization of the MCM DNA helicase at replication factories before the onset of DNA synthesis," Chromosoma, vol. 121, no. 5, pp. 499-507, 2012.

[60] M. A. Kuipers, T. J. Stasevich, T. Sasaki et al., "Highly stable loading of $\mathrm{Mcm}$ proteins onto chromatin in living cells requires replication to unload," The Journal of Cell Biology, vol. 192, no. 1, pp. 29-41, 2011.

[61] R. A. Laskey and M. A. Madine, "A rotary pumping model for helicase function of MCM proteins at a distance from replication forks," The EMBO Reports, vol. 4, no. 1, pp. 26-30, 2003.

[62] T. S. Takahashi, D. B. Wigley, and J. C. Walter, "Pumps, paradoxes and ploughshares: mechanism of the MCM2-7 DNA 
helicase," Trends in Biochemical Sciences, vol. 30, no. 8, pp. 437444, 2005.

[63] M. Mašata, P. Juda, O. Raška, M. C. Cardoso, and I. Raška, "A fraction of MCM 2 proteins remain associated with replication foci during a major part of S phase," Folia Biologica, vol. 57, no. 1, pp. 3-11, 2011.

[64] E. Guillou, A. Ibarra, V. Coulon et al., "Cohesin organizes chromatin loops at DNA replication factories," Genes and Development, vol. 24, no. 24, pp. 2812-2822, 2010.

[65] A. Nishiyama, L. Frappier, and M. Méchali, "MCM-BP regulates unloading of the MCM2-7 helicase in late S phase," Genes \& Development, vol. 25, no. 2, pp. 165-175, 2011.

[66] D. Cortez, G. Glick, and S. J. Elledge, "Minichromosome maintenance proteins are direct targets of the ATM and ATR checkpoint kinases," Proceedings of the National Academy of Sciences of the United States of America, vol. 101, no. 27, pp. 10078-10083, 2004.

[67] C.-C. Tsao, C. Geisen, and R. T. Abraham, "Interaction between human MCM7 and Rad17 proteins is required for replication checkpoint signaling," The EMBO Journal, vol. 23, no. 23, pp. 4660-4669, 2004.

[68] A. M. Woodward, T. Göhler, M. G. Luciani et al., "Excess Mcm27 license dormant origins of replication that can be used under conditions of replicative stress," The Journal of Cell Biology, vol. 173, no. 5, pp. 673-683, 2006.

[69] R. C. Alver, G. S. Chadha, and J. J. Blow, "The contribution of dormant origins to genome stability: from cell biology to human genetics," DNA Repair, vol. 19, no. 9, pp. 182-189, 2014.

[70] X. Q. Ge, D. A. Jackson, and J. J. Blow, “Dormant origins licensed by excess Mcm2-7 are required for human cells to survive replicative stress," Genes \& Development, vol. 21, no. 24, pp. 3331-3341, 2007.

[71] A. Ibarra, E. Schwob, and J. Méndez, "Excess MCM proteins protect human cells from replicative stress by licensing backup origins of replication," Proceedings of the National Academy of Sciences of the United States of America, vol. 105, no. 26, pp. 8956-8961, 2008.

[72] N. Shima, A. Alcaraz, I. Liachko et al., "A viable allele of Mcm4 causes chromosome instability and mammary adenocarcinomas in mice," Nature Genetics, vol. 39, no. 1, pp. 93-98, 2007.

[73] C.-H. Chuang, M. D. Wallace, C. Abratte, T. Southard, and J. C. Schimenti, "Incremental genetic perturbations to MCM2-7 expression and subcellular distribution reveal exquisite sensitivity of mice to DNA replication stress," PLoS Genetics, vol. 6, no. 9, Article ID e1001110, 2010.

[74] D. Kunnev, M. E. Rusiniak, A. Kudla, A. Freeland, G. K. Cady, and S. C. Pruitt, "DNA damage response and tumorigenesis in Mcm2-deficient mice," Oncogene, vol. 29, no. 25, pp. 3630-3638, 2010.

[75] K. Maki, T. Inoue, A. Onaka et al., "Abundance of prereplicative complexes (Pre-RCs) facilitates recombinational repair under replication stress in fission yeast," The Journal of Biological Chemistry, vol. 286, no. 48, pp. 41701-41710, 2011.

[76] J. J. Blow and A. Dutta, "Preventing re-replication of chromosomal DNA," Nature Reviews Molecular Cell Biology, vol. 6, no. 6, pp. 476-486, 2005.

[77] E. E. Arias and J. C. Walter, "Strength in numbers: preventing rereplication via multiple mechanisms in eukaryotic cells," Genes \& Development, vol. 21, no. 5, pp. 497-518, 2007.

[78] J. J. Blow, X. Q. Ge, and D. A. Jackson, "How dormant origins promote complete genome replication," Trends in Biochemical Sciences, vol. 36, no. 8, pp. 405-414, 2011.
[79] S. Ryu, J. Holzschuh, S. Erhardt, A.-K. Ettl, and W. Driever, "Depletion of minichromosome maintenance protein 5 in the zebrafish retina causes cell-cycle defect and apoptosis," Proceedings of the National Academy of Sciences of the United States of America, vol. 102, no. 51, pp. 18467-18472, 2005.

[80] C. R. Hughes, L. Guasti, E. Meimaridou et al., "MCM4 mutation causes adrenal failure, short stature, and natural killer cell deficiency in humans," The Journal of Clinical Investigation, vol. 122, no. 3, pp. 814-820, 2012.

[81] L. Gineau, C. Cognet, N. Kara et al., "Partial MCM4 deficiency in patients with growth retardation, adrenal insufficiency, and natural killer cell deficiency," The Journal of Clinical Investigation, vol. 122, no. 3, pp. 821-832, 2012.

[82] B. N. Bagley, T. M. Keane, V. I. Maklakova et al., "A dominantly acting murine allele of $\mathrm{Mcm} 4$ causes chromosomal abnormalities and promotes tumorigenesis," PLoS Genetics, vol. 8, no. 11, Article ID e1003034, 2012.

[83] S. C. Pruitt, K. J. Bailey, and A. Freeland, "Reduced Mcm2 expression results in severe stem/progenitor cell deficiency and cancer," Stem Cells, vol. 25, no. 12, pp. 3121-3132, 2007.

[84] M. E. Rusiniak, D. Kunnev, A. Freeland, G. K. Cady, and S. C. Pruitt, "Mcm2 deficiency results in short deletions allowing high resolution identification of genes contributing to lymphoblastic lymphoma," Oncogene, vol. 31, no. 36, pp. 4034-4044, 2012.

[85] B. Ren, G. Yu, G. C. Tseng et al., "MCM7 amplification and overexpression are associated with prostate cancer progression," Oncogene, vol. 25, no. 7, pp. 1090-1098, 2006.

[86] C. Giaginis, M. Georgiadou, K. Dimakopoulou et al., "Clinical significance of MCM-2 and MCM-5 expression in colon cancer: association with clinicopathological parameters and tumor proliferative capacity," Digestive Diseases and Sciences, vol. 54, no. 2, pp. 282-291, 2009.

[87] O. Saydam, O. Senol, T. B. M. Schaaij-Visser et al., "Comparative protein profiling reveals minichromosome maintenance (MCM) proteins as novel potential tumor markers for meningiomas," Journal of Proteome Research, vol. 9, no. 1, pp. 485-494, 2010.

[88] U. Cobanoglu, S. Mungan, C. Gundogdu, S. Ersoz, Y. Ozoran, and F. Aydin, "The expression of MCM-2 in invasive breast carcinoma: a stereologic approach," Bratislavské Lekárske Listy, vol. 111, no. 1, pp. 45-49, 2010.

[89] K.-M. Lau, Q. K. Y. Chan, J. C. S. Pang et al., "Minichromosome maintenance proteins 2, 3 and 7 in medulloblastoma: overexpression and involvement in regulation of cell migration and invasion," Oncogene, vol. 29, no. 40, pp. 5475-5489, 2010.

[90] C. Giaginis, A. Giagini, G. Tsourouflis et al., "MCM-2 and MCM-5 expression in gastric adenocarcinoma: clinical significance and comparison with Ki-67 proliferative marker," Digestive Diseases and Sciences, vol. 56, no. 3, pp. 777-785, 2011.

[91] A. F. Nicol, J. R. Lapa e Silva, C. B. Cunha et al., "Evaluation of MCM-2 expression in TMA cervical specimens," PLoS ONE, vol. 7, no. 4, Article ID e32936, 2012.

[92] M. Das, S. B. Prasad, S. S. Yadav et al., "Over expression of minichromosome maintenance genes is clinically correlated to cervical carcinogenesis," PLOS ONE, vol. 8, no. 7, Article ID e69607, 2013.

[93] M. L. Kuijjer, H. Rydbeck, S. H. Kresse et al., "Identification of osteosarcoma driver genes by integrative analysis of copy number and gene expression data," Genes Chromosomes and Cancer, vol. 51, no. 7, pp. 696-706, 2012. 
[94] X. Zhong, X. Chen, X. Guan et al., "Overexpressions of G9a and MCM7 in Esophageal squamous cell carcinoma associated with poor prognosis," Histopathology, 2014.

[95] A. Freeman, L. S. Morris, A. D. Mills et al., "Minichromosome maintenance proteins as biological markers of dysplasia and malignancy," Clinical Cancer Research, vol. 5, no. 8, pp. 21212132, 1999.

[96] S. B. Wharton, K. K. Chan, J. R. Anderson, K. Stoeber, and G. H. Williams, "Replicative $\mathrm{Mcm} 2$ protein as a novel proliferation marker in oligodendrogliomas and its relationship to Ki67 labelling index, histological grade and prognosis," Neuropathology and Applied Neurobiology, vol. 27, no. 4, pp. 305-313, 2001.

[97] K. Rodins, M. Cheale, N. Coleman, and S. B. Fox, "Minichromosome maintenance protein 2 expression in normal kidney and renal cell carcinomas: relationship to tumor dormancy and potential clinical utility," Clinical Cancer Research, vol. 8, no. 4, pp. 1075-1081, 2002.

[98] H. Kato, T. Miyazaki, Y. Fukai et al., "A new proliferation marker, minichromosome maintenance protein 2 , is associated with tumor aggressiveness in esophageal squamous cell carcinoma," Journal of Surgical Oncology, vol. 84, no. 1, pp. 24-30, 2003.

[99] P. Chatrath, I. S. Scott, L. S. Morris et al., "Aberrant expression of minichromosome maintenance protein-2 and Ki67 in laryngeal squamous epithelial lesions," British Journal of Cancer, vol. 89, no. 6, pp. 1048-1054, 2003.

[100] M. A. Gonzalez, S. E. Pinder, G. Callagy et al., "Minichromosome maintenance protein 2 is a strong independent prognostic marker in breast cancer," Journal of Clinical Oncology, vol. 21, no. 23, pp. 4306-4313, 2003.

[101] C. Kobierzycki, B. Pula, A. Wojnar, M. Podhorska-Okolow, and P. Dziegiel, "Tissue microarray technique in evaluation of proliferative activity in invasive ductal breast cancer," Anticancer Research, vol. 32, no. 3, pp. 773-777, 2012.

[102] E. C. Obermann, P. Went, A. Zimpfer et al., "Expression of minichromosome maintenance protein 2 as a marker for proliferation and prognosis in diffuse large B-cell lymphoma: a tissue microarray and clinico-pathological analysis," BMC Cancer, vol. 5, article 162, 2005.

[103] J. Szelachowska, P. Dziegiel, J. Jelen-Krzeszewska et al., "Mcm-2 protein expression predicts prognosis better than Ki-67 antigen in oral cavity squamocellular carcinoma," Anticancer Research, vol. 26, no. 3, pp. 2473-2478, 2006.

[104] H. Gakiopoulou, P. Korkolopoulou, G. Levidou et al., "Minichromosome maintenance proteins 2 and 5 in non-benign epithelial ovarian tumours: relationship with cell cycle regulators and prognostic implications," British Journal of Cancer, vol. 97, no. 8, pp. 1124-1134, 2007.

[105] M. Liu, J.-S. Li, D.-P. Tian, B. Huang, S. Rosqvist, and M. Su, "MCM2 expression levels predict diagnosis and prognosis in gastric cardiac cancer," Histology and Histopathology, vol. 28, no. 4, pp. 481-492, 2013.

[106] Y. S. Lee, S.-A. Ha, H. J. Kim et al., "Minichromosome maintenance protein 3 is a candidate proliferation marker in papillary thyroid carcinoma," Experimental and Molecular Pathology, vol. 88, no. 1, pp. 138-142, 2010.

[107] J. Kikuchi, I. Kinoshita, Y. Shimizu et al., "Minichromosome maintenance (MCM) protein 4 as a marker for proliferation and its clinical and clinicopathological significance in non-small cell lung cancer," Lung Cancer, vol. 72, no. 2, pp. 229-237, 2011.

[108] T. J. Dudderidge, J. D. Kelly, A. Wollenschlaeger et al., "Diagnosis of prostate cancer by detection of minichromosome maintenance 5 protein in urine sediments," The British Journal of Cancer, vol. 103, no. 5, pp. 701-707, 2010.

[109] T. Zheng, M. Chen, S. Han et al., "Plasma minichromosome maintenance complex component 6 is a novel biomarker for hepatocellular carcinoma patients," Hepatology Research, 2014.

[110] K. Nishihara, K. Shomori, S. Fujioka et al., "Minichromosome maintenance protein 7 in colorectal cancer: implication of prognostic significance," International Journal of Oncology, vol. 33, no. 2, pp. 245-251, 2008.

[111] S. Fujioka, K. Shomori, K. Nishihara et al., "Expression of minichromosome maintenance 7 (MCM7) in small lung adenocarcinomas (pT1): prognostic implication," Lung Cancer, vol. 65, no. 2, pp. 223-229, 2009.

[112] T. Tamura, K. Shomori, T. Haruki et al., "Minichromosome maintenance-7 and geminin are reliable prognostic markers in patients with oral squamous cell carcinoma: Immunohistochemical study," Journal of Oral Pathology and Medicine, vol. 39, no. 4, pp. 328-334, 2010.

[113] J. J. Blow and B. Hodgson, "Replication licensing-defining the proliferative state?” Trends in Cell Biology, vol. 12, no. 2, pp. 72$78,2002$.

[114] J. J. Blow and P. J. Gillespie, "Replication licensing and cancera fatal entanglement?" Nature Reviews Cancer, vol. 8, no. 10, pp. 799-806, 2008.

[115] O. J. Kayes, M. Loddo, N. Patel et al., "DNA replication licensing factors and aneuploidy are linked to tumor cell cycle state and clinical outcome in penile carcinoma," Clinical Cancer Research, vol. 15, no. 23, pp. 7335-7344, 2009.

[116] C. P. Harris, X. Y. Lu, G. Narayan, B. Singh, V. V. V. S. Murty, and P. H. Rao, "Comprehensive molecular cytogenetic characterization of cervical cancer cell lines," Genes Chromosomes and Cancer, vol. 36, no. 3, pp. 233-241, 2003.

[117] P. H. Rao, H. Arias-Pulido, X.-Y. Lu et al., "Chromosomal amplifications, $3 \mathrm{q}$ gain and deletions of $2 \mathrm{q} 33-\mathrm{q} 37$ are the frequent genetic changes incervical carcinoma," BMC Cancer, vol. 4, article 5, 2004.

[118] G. Narayan, V. Bourdon, S. Chaganti et al., "Gene dosage alterations revealed by cDNA microarray analysis in cervical cancer: identification of candidate amplified and overexpressed genes," Genes Chromosomes and Cancer, vol. 46, no. 4, pp. 373-384, 2007.

[119] T. A. Potapova, J. Zhu, and R. Li, "Aneuploidy and chromosomal instability: a vicious cycle driving cellular evolution and cancer genome chaos," Cancer and Metastasis Reviews, vol. 32, no. 3-4, pp. 377-389, 2013.

[120] S. Shreeram, A. Sparks, D. P. Lane, and J. J. Blow, "Cell typespecific responses of human cells to inhibition of replication licensing," Oncogene, vol. 21, no. 43, pp. 6624-6632, 2002.

[121] D. Feng, Z. Tu, W. Wu, and C. Liang, "Inhibiting the expression of DNA replication-initiation proteins induces apoptosis in human cancer cells," Cancer Research, vol. 63, no. 21, pp. 73567364, 2003.

[122] Y. Liu, G. He, Y. Wang, X. Guan, X. Pang, and B. Zhang, "MCM2 is a therapeutic target of Trichostatin A in colon cancer cells," Toxicology Letters, vol. 221, no. 1, pp. 23-30, 2013.

[123] E. P. Erkan, T. Ströbel, G. Lewandrowski et al., "Depletion of minichromosome maintenance protein 7 inhibits glioblastoma multiforme tumor growth in vivo," Oncogene, vol. 33, pp. 47784785, 2013.

[124] T. J. Dudderidge, S. R. McCracken, M. Loddo et al., "Mitogenic growth signalling, DNA replication licensing, and survival are 
linked in prostate cancer," British Journal of Cancer, vol. 96, no. 9, pp. 1384-1393, 2007.

[125] T. Valovka, M. Schönfeld, P. Raffeiner et al., "Transcriptional control of DNA replication licensing by Myc," Scientific Reports, vol. 3, article 3444, 2013.

[126] S. Majid, A. A. Dar, S. Saini et al., "Regulation of minichromosome maintenance gene family by MicroRNA-1296 and genistein in prostate cancer," Cancer Research, vol. 70, no. 7, pp. 2809-2818, 2010.

[127] J. H. Luo, "Oncogenic activity of MCM7 transforming cluster," World Journal of Clinical Oncology, vol. 2, no. 2, pp. 120-124, 2011.

[128] K. Yankulov, I. Todorov, P. Romanowski et al., "MCM proteins are associated with RNA polymerase II holoenzyme," Molecular and Cellular Biology, vol. 19, no. 9, pp. 6154-6163, 1999.

[129] M. Snyder, W. He, and J. J. Zhang, “The DNA replication factor MCM5 is essential for Statl-mediated transcriptional activation," Proceedings of the National Academy of Sciences of the United States of America, vol. 102, no. 41, pp. 14539-14544, 2005.

[130] M. E. Hubbi, W. Luo, J. H. Baek, and G. L. Semenza, "MCM proteins are negative regulators of hypoxia-inducible factor 1 ," Molecular Cell, vol. 42, no. 5, pp. 700-712, 2011.

[131] I. M. Slaymaker, Y. Fu, D. B. Toso et al., "Mini-chromosome maintenance complexes form a filament to remodel DNA structure and topology," Nucleic Acids Research, vol. 41, no. 5, pp. 3446-3456, 2013. 

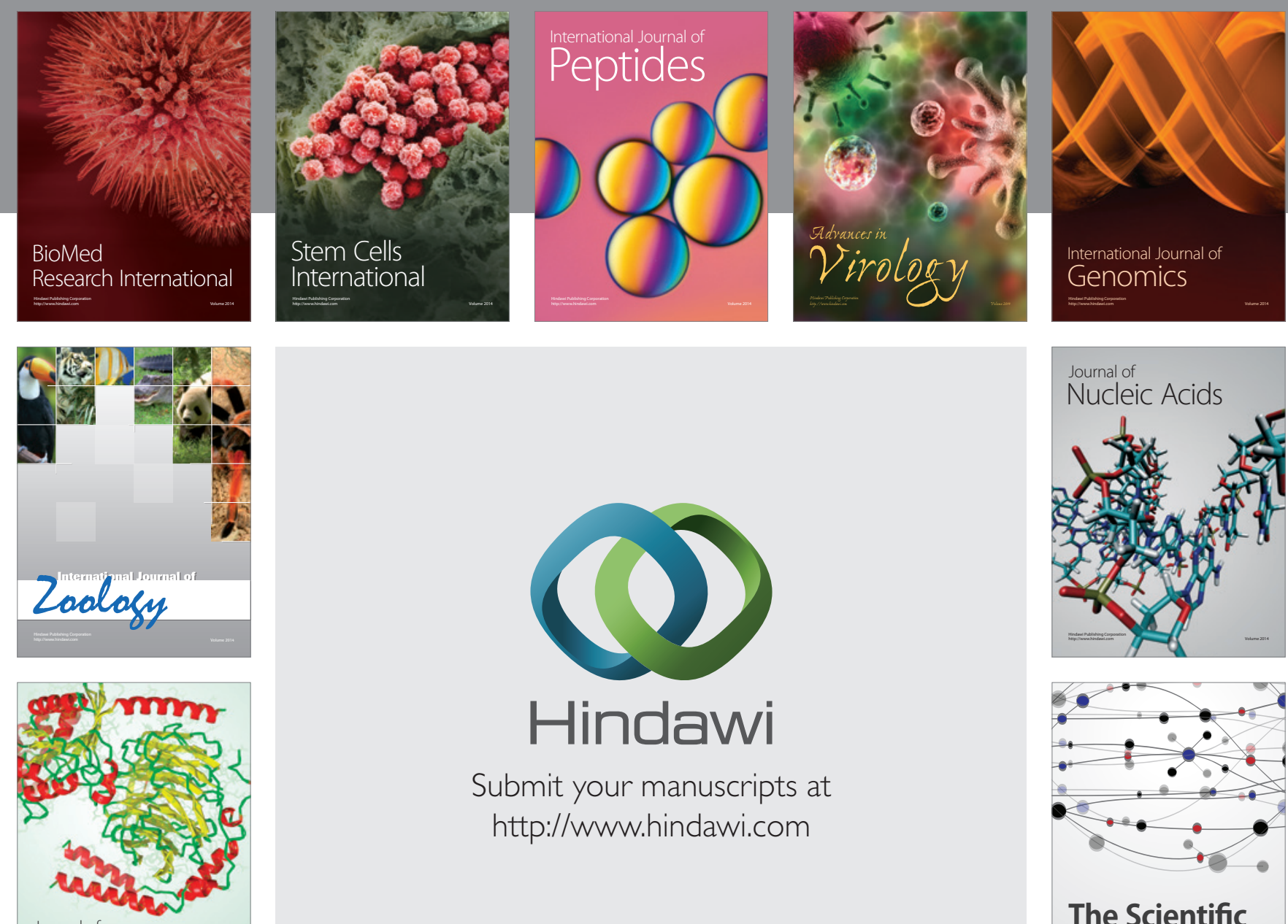

Submit your manuscripts at

http://www.hindawi.com

Journal of
Signal Transduction
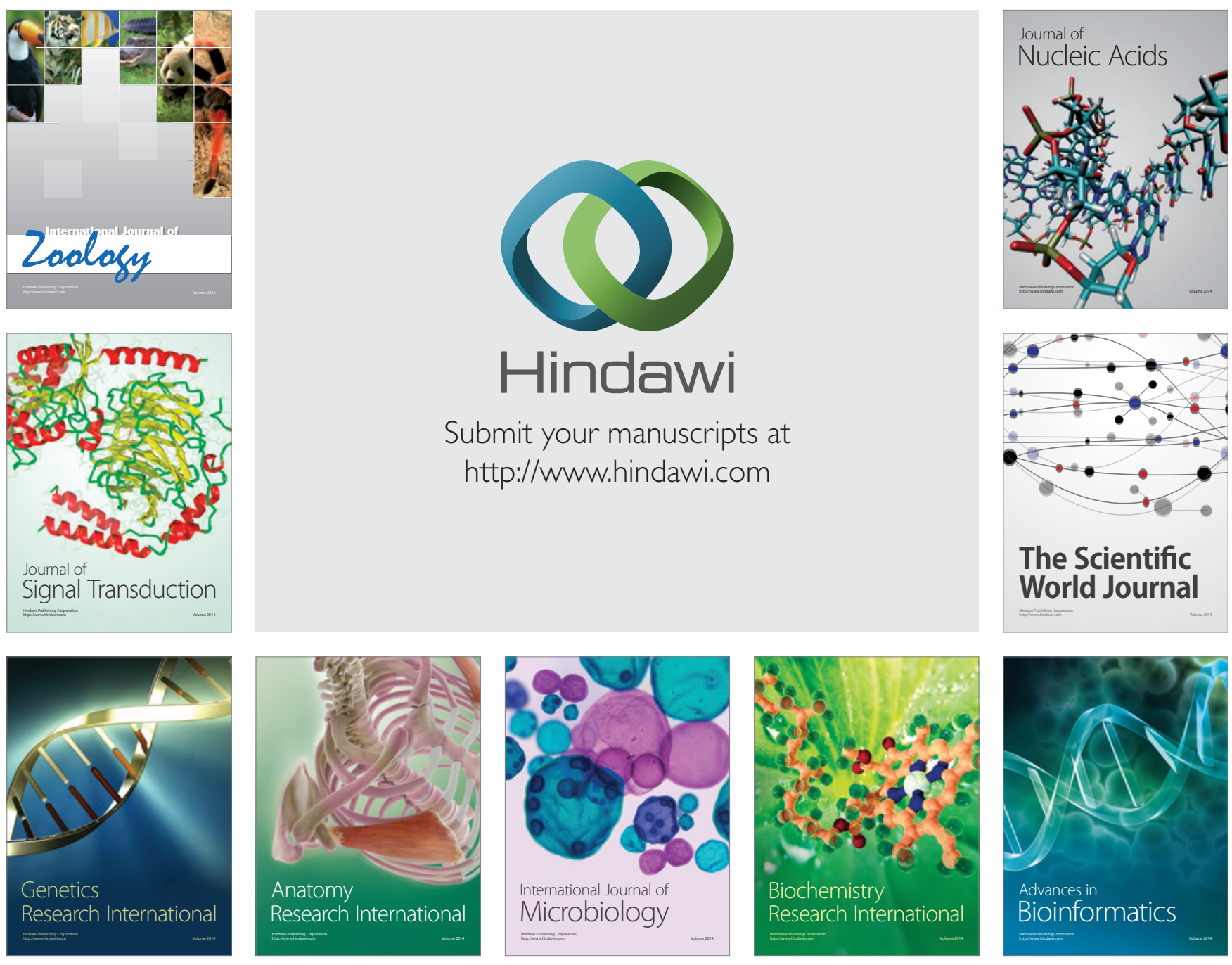

The Scientific World Journal
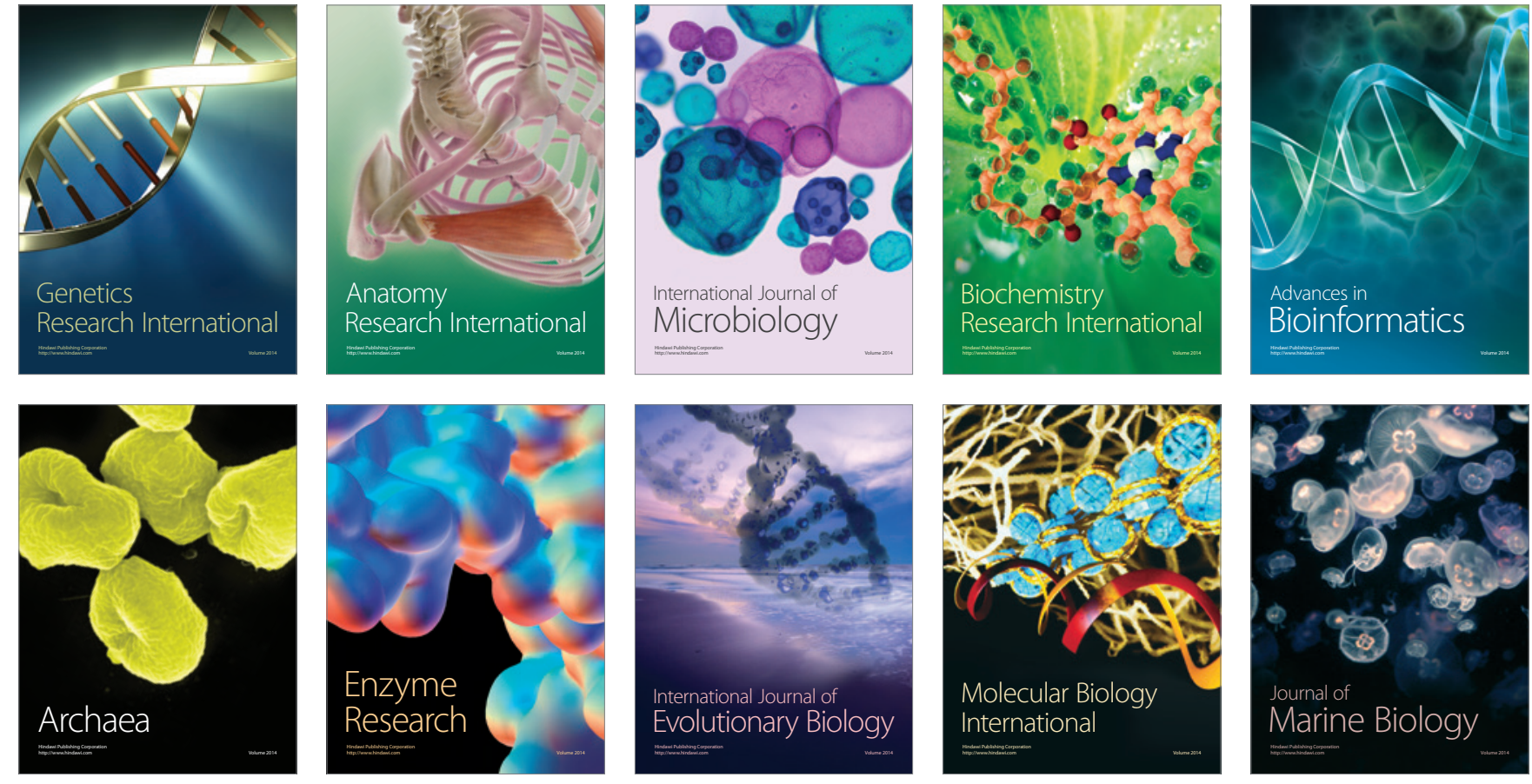